\title{
Differential regional expression of brain-derived neurotrophic factor following olfactory fear learning
}

\author{
Seth V. Jones, Lisa Stanek-Rattiner, Michael Davis, and Kerry J. Ressler ${ }^{1}$ \\ Department of Psychiatry and Behavioral Sciences, Yerkes Research Center, Emory University, Atlanta, Georgia 30329, USA
}

\begin{abstract}
We examined brain-derived neurotrophic factor (BDNF) mRNA expression across the olfactory system following fear conditioning. Mice received 10 pairings of odor with footshock or equivalent unpaired odors and shocks. We found increased BDNF mRNA in animals receiving paired footshocks in the multiple regions examined including the posterior piriform cortex (PPC) and basolateral amygdala (BLA). This was in contrast to the unpaired and odor-alone treatments, where BDNF mRNA was increased in the olfactory bulb (OB) and anterior piriform cortex (APC) only, but not the higher olfactory areas. We propose that odor exposure increases expression of BDNF in the OB and APC while the PPC and BLA increase BDNF mRNA only when associative learning occurs.
\end{abstract}

Brain-derived neurotrophic factor (BDNF) is a member of the neurotrophin family that has been shown to play a role in learning and memory (Tyler et al. 2002; Rattiner et al. 2005). Besides its neurotrophic activity, BDNF, working through its primary receptor TrkB, is involved in a number of downstream effects related to learning including long-term synaptic plasticity (Lohof et al. 1993; Korte et al. 1995), morphological changes in dendritic spines (Tyler and Pozzo-Miller 2003; Rex et al. 2007), gene transcription via CREB (Minichiello et al. 2002), and the recruitment of PSD-95 to the synapse (Yoshii and Constantine-Paton 2007).

In the hippocampus, BDNF mRNA increases with hippocampal-dependent tasks such as the Morris Water Maze (Falkenberg et al. 1992) and contextual learning (Hall et al. 2000; Mizuno et al. 2000). Locally blocking the action of BDNF prevents learning in hippocampal-dependent tasks as shown by infusion of antisense BDNF oligonucleotides (Mizuno et al. 2000) and by regional knock-out of BDNF (Heldt et al. 2007). In the amygdala, expression of a truncated form of the TrkB receptor, which blocks the activity of the full-length receptor, blocks both the acquisition of a cued fear (Rattiner et al. 2004b) and extinction of fear (Chhatwal et al. 2006).

Although many studies of BDNF in sensory systems have focused on its developmental role, few studies have linked BDNF to learning in the olfactory system, even though the olfactory system at multiple levels shows signs of learning-induced change, including LTP (Ennis et al. 1998; Lebel et al. 2001), dendritic spine plasticity (Knafo et al. 2001), and c-fos activation (Funk and Amir 2000; Schettino and Otto 2001; Illig 2007). In the olfactory system, BDNF is expressed in the olfactory bulb and piriform cortex, and is upregulated in these areas following kainic acid-induced seizures (Katoh-Semba et al. 1999). In slice preparations of the main olfactory bulb, BDNF has been shown to cause an increase in dendritic spines in granule cells (Berghuis et al. 2006). In the piriform cortex, deficits in BDNF have been linked to a decrease in dendritic spine number (Nanobashvili et al. 2005). BDNF is also essential to the differentiation of regenerating cells within the olfactory bulb (Benraiss et al. 2001).

No study has yet investigated a role for learning-related BDNF across the adult olfactory system. We predicted that BDNF would be dynamically regulated in the different areas of the olfactory system during an olfactory-cued learning task. For our

\footnotetext{
1 Corresponding author.
}

E-mail kressle@emory.edu; fax (404) 727-8070.

Article is online at http://www.learnmem.org/cgi/doi/10.1101//m.781507. experiments, we made use of olfactory-cued fear conditioning, a form of classical conditioning that induces rapid and robust learning in rodent models (Richardson et al. 1999; Paschall and Davis 2002; Jones et al. 2005).

In these experiments, we measured BDNF mRNA at four different levels within the olfactory system: the mitral cell layer (M) of the main olfactory bulb (OB) (Fig. 1, left), the anterior piriform cortex (APC; Fig. 1 center), the posterior piriform cortex (PPC), and the basolateral amygdala (BLA) (Fig. 1, right). Hybridization density of the RNA probes was quantified using the mean luminosity histogram function in Adobe Photoshop. For each slide, the best anatomically defined section was selected and the luminosity of the region of interest (ROI: OB, APC, PPC, or BLA) was quantified, while a second ROI of the exact same shape directly adjacent to this region but in an area of background staining was also quantified (background ROI: OB, external plexiform layer; APC, cortical layer 1, and lateral olfactory tract; PPC /BLA, cortical layer 1 and internal capsule). For both the right and left sides for each section separately, the background ROI was subtracted from the ROI to provide a normalized luminosity level for each area. The linear nature of this method of quantification compared to other quantification methods have been previously examined in our lab (Rattiner et al. 2004a,b; Chhatwal et al. 2006; Heldt et al. 2007).

Throughout all experiments, we followed a training procedure similar to those more fully described previously (Jones et al. 2005). All animals were adult (8-12 wk) male C57Bl/6J mice (Jackson Laboratories) in standard group housing ( $\leq 5 /$ cage), were given ad libitum access to food and water, on a 12-h on/ 12-h off light cycle. All experiments were performed during the light cycle. All experiments were performed as approved by the Emory University Institutional Review Board and NIH IACUC standards. For the odor stimulus, mixtures of 5\% volume/volume amyl acetate (Sigma-Aldrich) were dissolved in propylene glycol (Sigma-Aldrich) and attached to the odor training apparatus.

Before each experiment, mice were given $3 \mathrm{~d}$ of preexposure to the startle chambers to acclimate the animals to handling. Prior to conditioning the animals first received 15 startle stimulus presentations to habituate startle to a stable baseline level. The following day the animals received a preconditioning test (pretest), consisting of 10 odor-startle trials intermingled with 10 startle-alone trials. The startle stimulus was a $50-\mathrm{msec}$ burst of 105-dB white noise. Startle was measured by peak amplitude of cage displacement during the $150 \mathrm{msec}$ following startle stimulus onset. 


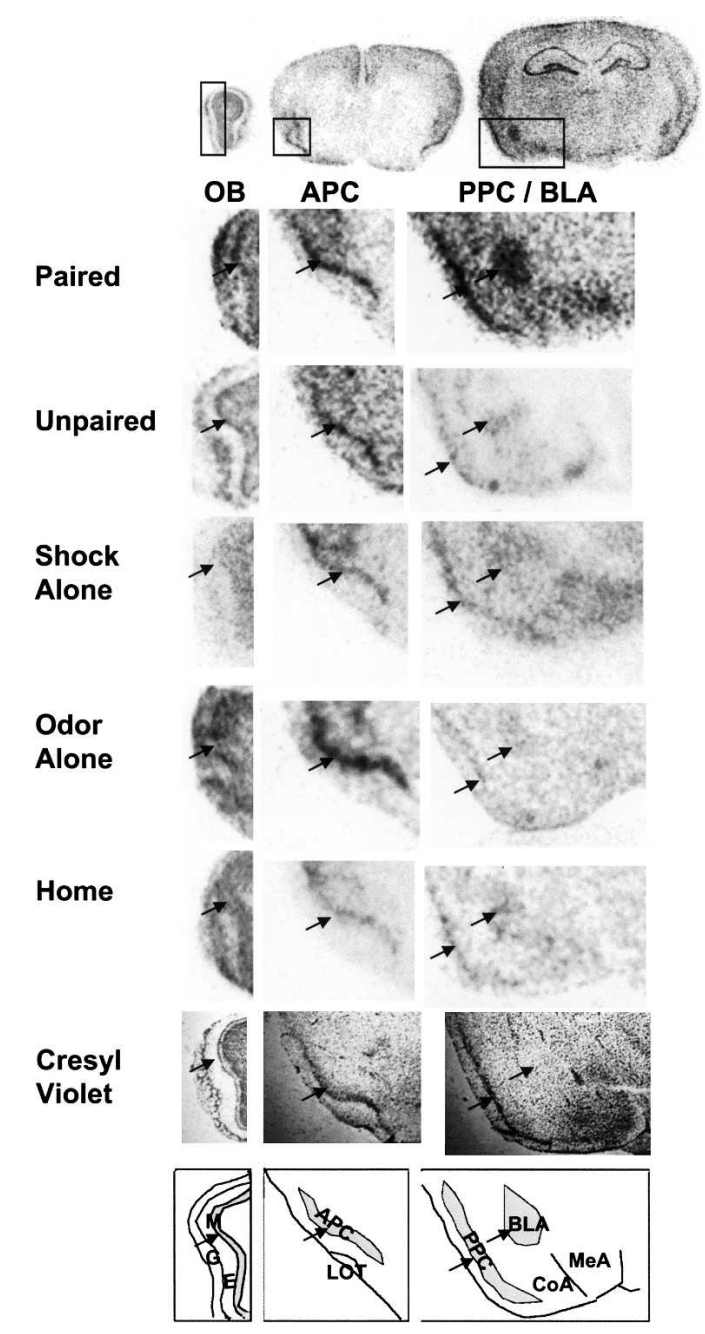

Figure 1. Sections from the main olfactory bulb, anterior piriform cortex, and the posterior piriform cortex and basolateral amygdala. The mitral cell layer was defined as the best-defined layer interior to the external plexiform layer based on cellular architecture (cresyl violet; bottom). Because the piriform cortex reaches extensively anterior to posterior, care was taken to define markers for the anterior and posterior piriform. Measurements for the anterior piriform were taken from the most posterior section that still showed the curve of the anterior pirifom cortex around the lateral olfactory tract. Posterior piriform cortex measurements were taken from the section that included the ventromedial hypothalamus. For each region, selection of the region of interest was confirmed by cresyl violet staining (bottom), and is illustrated by arrows. Absolute luminosity levels from the in situ autoradiogram from exemplary regions of interest (ROls; the shaded areas in the bottom drawing) are subtracted from nonspecific background for each area as described in text. Key: G, glomerular layer; E, external plexiform layer; M, mitral cell layer; APC, anterior piriform cortex; LOT, lateral olfactory tract; PIR, posterior piriform cortex; BLA, basolateral amygdala complex; CoA, cortical amygala; MeA, medial amygdala.

In the first experiment, we paired amyl acetate with a 0.25 sec, 0.4-mA footshock for one group of animals $(n=16)$. Other groups $(n=8)$ were exposed to amyl acetate and shock in an unpaired fashion or shock only with no odor. During training, freezing was assessed for all groups except the home cage group (Fig. 2A). Activity was measured during a 5-sec "window" starting $2 \mathrm{sec}$ after odor onset, or in the shockalone group, 8 sec before the shock. Freezing was assessed by analyzing the cage displacement during the 5-sec activity window. A repeated-measures ANOVA showed a significant group by trial interaction over the five trials $\left(F_{(10,24)}=3.081\right.$, $P<0.01)$. A significant interaction for trial was also shown by repeated-measures ANOVA $\left(F_{(5,24)}=8.081, P<0.01\right)$. Post-hoc LSD pairwise comparisons showed that freezing significantly increased from pretest to the final freezing measurement for both the paired and shock-alone groups $(P<0.05)$. Note that an increase in freezing during exposure to shock without odor is not unexpected, as this group would be expected to undergo some amount of contextual freezing during the training period.

Prior work in our lab found that BDNF mRNA induction was maximal $2 \mathrm{~h}$ after fear conditioning (Rattiner et al. 2004b). Thus, $2 \mathrm{~h}$ following training, half of the paired group and all of the other groups were sacrificed by chloral hydrate overdose and their brains were removed and frozen on dry ice. The other half of the odor-shock paired group was tested for fear-potentiated startle the following day. Brains were sliced at 30-micron sections with a cryostatic microtome at $-20^{\circ} \mathrm{C}$ onto SuperFrost Plus slides. Each slide contained multiple sections from the olfactory bulb, anterior piriform cortex, and posterior piriform/amygdala anatomical areas. Slides were stored at $-80^{\circ} \mathrm{C}$ until prepared for hybridization.

A clone was created for the full coding sequence (Exon V) of BDNF from the NIH database IMAGE \#1851120, GenBank \#3837569. This probe was previously validated and described (Rattiner et al. 2004a), and the in situ hybridization procedure is the same as in that manuscript. Briefly, radioactive antisense riboprobes were created using T7 polymerase using ${ }^{35} \mathrm{~S}$-UTP, and hybridizations were performed at $50^{\circ} \mathrm{C}$ overnight. Following stringent washes, slides were placed against Kodak MR audioradiography film. Films were developed after $3 \mathrm{~d}$ of exposure. Previous parametric studies in our lab have demonstrated the specificity of the BDNF antisense compared to sense riboprobes, the ability of excess cold antisense message to compete with the radioactive antisense riboprobe (Rattiner et al. 2004a,b; Heldt et al. 2007).

Figure 1 shows qualitative levels of expression of BDNF mRNA levels in the OB, APC, PPC, and BLA among paired, unpaired, and shock-alone groups compared to home cage (Fig. 2B). A significant between-groups effect was found for BDNF mRNA expression in the OB (Fig. 2B; Table 1) (ANOVA, $F_{(3,24)}=3.377$, $P<0.05)$. Both paired and unpaired groups expressed significantly more BDNF mRNA than did the home cage group (posthoc $P<0.05$ ). The shock-alone group showed no significant difference from home cage. Although a one-way ANOVA of the anterior piriform cortex (APC) did not show a significant difference for any group $\left(F_{(3,25)}=1.972, P>0.05\right)$, planned post-hoc $t$-tests showed significant differences from home cage controls in the paired and unpaired groups $(\mathrm{P}<0.05)$ (Fig. $2 \mathrm{~B})$. In both the PPC (ANOVA, $F_{(3,25)}=6.930, P<0.05$ ), and BLA (ANOVA, $\left.F_{(3,28)}=5.131, P<0.05\right)$, there was a main effect for group. In both regions, post-hoc LSD tests showed only the paired group was significantly different from home cage $(P<0.05)$ (Fig. $2 \mathrm{~B})$. These data suggest that only groups receiving associative olfactory conditioning had increased BDNF mRNA in the BLA and PPC, whereas simply exposure to odor led to increases in BDNF mRNA in both the OB and APC. Furthermore, experiencing the same level of stress via a shock without an odor exposure, did not lead to significant induction of BDNF mRNA across any of these olfactory areas.

In the second experiment, two different groups received odor-shock paired or unpaired training while a third group received amyl acetate odor alone to examine if odor exposure alone, in the absence of any shock, would lead to increases in BDNF expression in the OB and APC. Freezing was again measured during training (Fig. 3A). A significant group by 
A

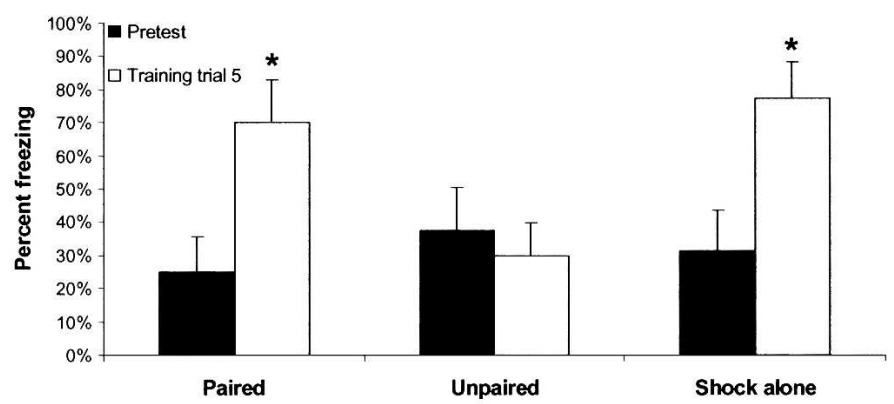

B

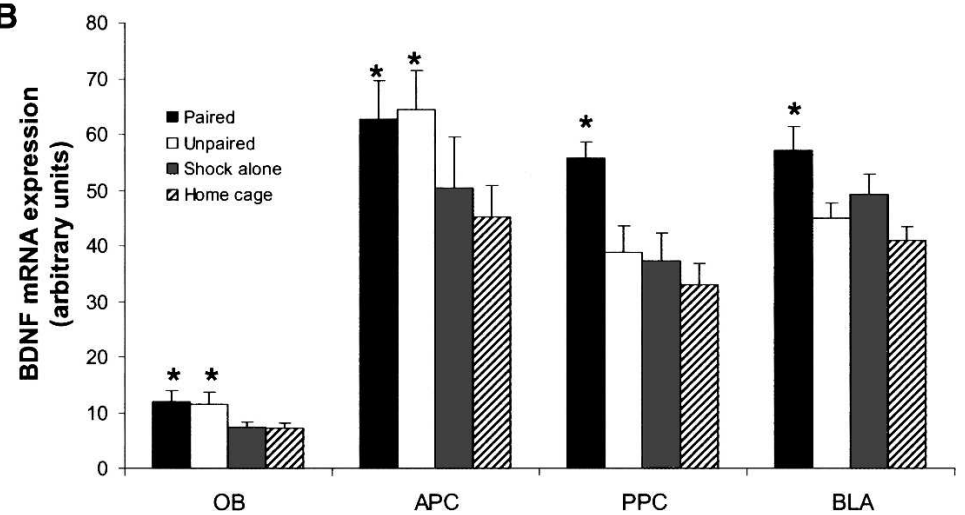

Figure 2. Experiment 1. (A) Amount of freezing during the training session is shown for all groups. Freezing increases during training for paired and shock-alone groups. Asterisks represent significant $(P<0.05)$ pairwise post-hoc tests between each trial and pretraining freezing. $(B)$ Level of BDNF mRNA expression is shown for each area and each experimental group (based on autoradiogram signal density, in arbitrary units of luminosity). Radiolabeled probe signal density for each anatomical area was quantified in relation to background signal from low-expressing adjacent regions. Asterisks represent significant $(P<0.05)$ difference from home cage in pairwise post-hoc tests. Bars illustrate mean \pm SEM.

trial interaction was revealed by repeated-measures ANOVA $\left(F_{(10,24)}=4.529, P<0.01\right)$. As before, animals that had odor and shock paired showed an increase in freezing by post-hoc LSD test $(P<0.05)$. Neither odor-alone nor unpaired groups showed an increase in freezing (Fig. 3A).

BDNF mRNA expression was significantly different from home cage controls in the OB in the odor-alone, paired odorshock, and unpaired odor-shock groups (Fig. 3B; Table 2) $\left(F_{(3,32)}=4.290, P<0.05\right)$. Post-hoc LSD tests showed a significant difference from home cage for all three groups $(P<0.05)$. As in the previous experiment, the APC did not show a significant main effect of group $\left(F_{(3,32)}=2.137, P=0.12\right)$. In the PPC, a main effect for group was found $\left(F_{(3,32)}=3.106, P<0.05\right)$. Post-hoc LSD tests showed that only the paired group was significantly different from home cage control $(P<0.05)$ (Fig. 3B). In the BLA, a main effect for group was also found $\left(F_{(3,32)}=3.173, P<0.05\right)$. The paired group showed a significant difference in post-hoc LSD tests from each of the other groups $(P<0.05)$. The fact that the odor-only group had equivalent expression of BDNF mRNA in primary sensory areas suggests that exposure to odor alone was sufficient for its induction, rather than the stress of shock. The striking finding that BDNF mRNA expression was elevated in the primary sensory areas following odor exposure, whereas it was elevated in the higher associative areas only after odor-shock pairings also replicated the main findings of the first experiment.

Because the paired and unpaired groups for both experiments were exposed to both odors and shocks, but only the paired group showed an increase in freezing to the odor, we used the freezing data from these two groups to examine how the levels of BDNF mRNA correlate with freezing behavior. No correlation was seen between mRNA expression of BDNF and freezing in the OB ( $\mathrm{n}=26$, Pearson's correlation coefficient $=0.03, P>0.05)$. The APC also showed no correlation between freezing behavior and BDNF mRNA expression $(\mathrm{n}=29$, Pearson's correlation coefficient $=-0.01, P>0.05)$. In contrast, a weak, but significant positive correlation between freezing and BDNF mRNA expression was found in the PPC $(\mathrm{n}=29$, Pearson's correlation coefficient $=0.52$, $P<0.05)$, as well as in the BLA ( $\mathrm{n}=30$, Pearson's correlation coefficient $=0.38$, $P<0.05)$. Thus, BDNF expression was correlated with level of fear learning in areas involved in olfactory-shock associations, but not in the more proximal primary olfactory areas.

These experiments are the first to show a role for BDNF across the olfactory system in a learning paradigm. The results expand on prior observations showing that BDNF mRNA is upregulated in areas associated with a certain task, such as the amygdala during fear learning, and the hippocampus during spatial learning. These results also show that BDNF is differentially expressed in anterior versus posterior regions of the olfactory system with odor exposure and fear learning.

The mitral cell layer of the olfactory bulb $(\mathrm{OB})$ and the anterior piriform cortex (APC) showed similar levels of BDNF mRNA in both odorshock paired and unpaired groups in the first experiment (Fig. 2B). In the second experiment, odor-alone, odor-shock unpaired, and odor-shock paired groups showed similar levels of BDNF message in the OB and APC (Fig. 3B). BDNF mRNA did not correlate with fear as measured by freezing at the end of the session in either the OB or APC. The posterior piriform cortex (PPC) and basolateral amygdala (BLA) showed increased BDNF mRNA in the odor-shock paired group, but not the unpaired, odor-alone, or shock-alone groups. In both of these areas, BDNF mRNA expression was significantly, albeit weakly, correlated with freezing at the end of the session.

Thus, BDNF mRNA in the OB and APC seems to be induced simply by exposure to olfactory stimuli, regardless of whether the odor is associated with the shock. In contrast, BDNF mRNA is only induced in the PPC and BLA when there is an association

Table 1. Absolute values (mean \pm SEM) of mRNA expression (autoradiogram signal intensities) for Experiment 1

\begin{tabular}{lcccc}
\hline & OB & \multicolumn{1}{c}{ APIR } & \multicolumn{1}{c}{ PPIR } & BLA \\
\hline Paired & $11.8 \pm 2.2^{*}$ & $62.8 \pm 6.7^{*}$ & $55.6 \pm 3.0^{*}$ & $57.1 \pm 4.3^{*}$ \\
Unpaired & $11.5 \pm 2.1^{*}$ & $64.4 \pm 7.0^{*}$ & $38.9 \pm 4.6$ & $44.9 \pm 2.8$ \\
Shock & $7.5 \pm 0.9$ & $50.4 \pm 9.1$ & $37.4 \pm 4.9$ & $49.2 \pm 3.5$ \\
Home & $7.5 \pm 0.9$ & $45.1 \pm 5.6$ & $33.1 \pm 3.6$ & $44.0 \pm 2.3$ \\
\hline
\end{tabular}

Asterisks represent significant $(P \leq 0.05)$ difference from home cage. 
A

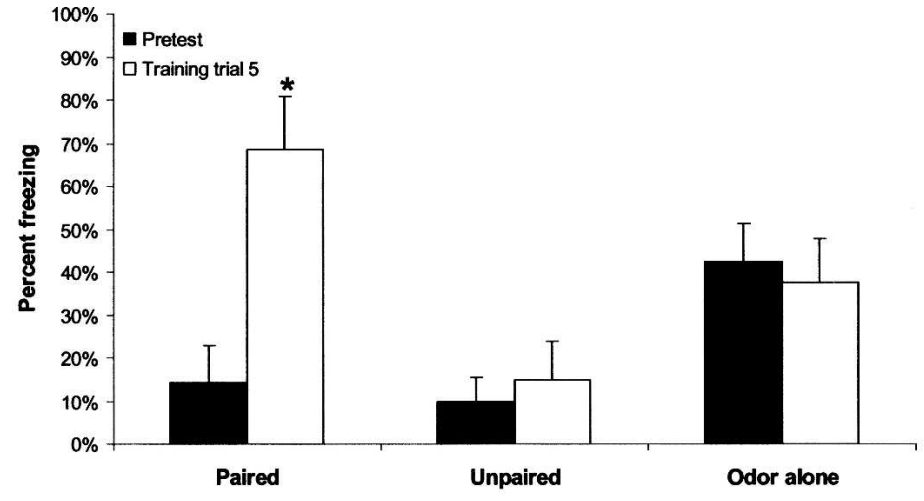

B

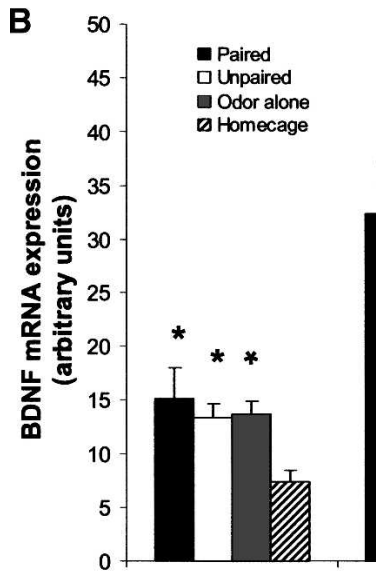

$\mathrm{OB}$

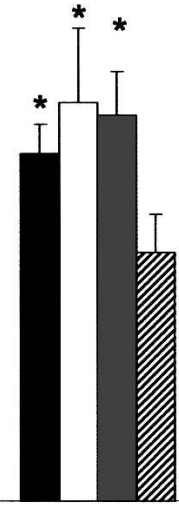

APC

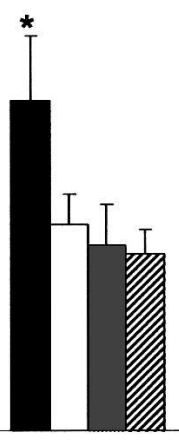

PPC

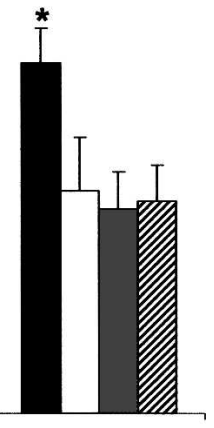

BLA

Figure 3. Experiment 2. (A) Amount of freezing during the training session is shown for all groups. Freezing increases during training for the paired group but not the unpaired or odor-alone groups. Asterisks represent significant $(P<0.05)$ pairwise post-hoc tests between each trial and pretraining freezing. (B) Level of BDNF mRNA expression is shown for each area and each experimental group (based on autoradiogram signal density, in arbitrary units of luminosity). Signals were quantified as in Figure 1. Asterisks represent significant $(P<0.05)$ difference from home cage in pairwise post-hoc tests. Bars illustrate mean \pm SEM.

between odor and shock to support learning. This division suggests, at least with respect to BDNF mRNA and fear conditioning, that the OB and APC are mainly involved in odor identification, while the PPC and BLA are involved in associative learning, probably because they receive broad input from cortical areas outside the olfactory system, including shock inputs required for learning to fear the odor (Johnson et al. 2000).

The increase in BDNF mRNA with nonassociative presentations of stimuli does not preclude the idea that BDNF is a marker for synaptic plasticity and learning. Mitral cell receptive fields can grow more specific after repeated odor exposure (Fletcher and Wilson 2003). Likewise, with experience, APC neurons increase in their ability to discriminate between similar odors (Wilson 2003). Thus, the increases in BDNF mRNA that we observed

Table 2. Absolute values (mean \pm SEM) of mRNA expression (autoradiogram signal intensities) for Experiment 2

\begin{tabular}{lcccc}
\hline & OB & \multicolumn{1}{c}{ APIR } & \multicolumn{1}{c}{ PPIR } & BLA \\
\hline Paired & $15.2 \pm 2.8^{*}$ & $32.3 \pm 2.7^{*}$ & $30.8 \pm 6.0^{*}$ & $32.7 \pm 3.2^{*}$ \\
Unpaired & $13.3 \pm 1.3^{*}$ & $37.1 \pm 6.9^{*}$ & $19.2 \pm 2.9$ & $20.8 \pm 5.1$ \\
Odor & $13.8 \pm 1.2^{*}$ & $35.9 \pm 4.1^{*}$ & $17.2 \pm 3.8$ & $19.1 \pm 3.4$ \\
Home & $7.4 \pm 1.8$ & $23.1 \pm 3.6$ & $16.5 \pm 2.3$ & $19.8 \pm 3.4$ \\
\hline
\end{tabular}

Asterisks represent significant $(P \leq 0.05)$ difference from home cage. in the OB and APC in response to odor presentation could reflect learning not indicative of odor-shock association taking place in these areas. If so, BDNF may also be involved in nonassociative olfactory learning. It has previously been shown within the olfactory system that acute application of BDNF causes tyrosine phosphorylation-induced suppression of the voltage-gated potassium channel Kv1.3 in the olfactory bulb (Tucker and Fadool 2002). This is particularly intriguing because of the increase in olfactory acuity with a Kv1.3 deletion, thus linking BDNF to olfactory acuity (Fadool et al. 2004). Further studies of BDNF in the olfactory system should clarify the causal role of these downstream effects in olfactory learning.

\section{Acknowledgments}

Support was provided by NIH (MH069884, DA-019624 to K.J.R., R37 MH47840 to M.D.), NARSAD, the Center for Behavioral Neuroscience (NSF agreement IBN-987675), Burroughs Wellcome Fund, and by an NIH/NCRR base grant (P51RR000165) to Yerkes National Primate Research Center.

\section{References}

Benraiss, A., Chmielnicki, E., Lerner, K., Roh, D., and Goldman, S.A. 2001. Adenoviral brain-derived neurotrophic factor induces both neostriatal and olfactory neuronal recruitment from endogenous progenitor cells in the adult forebrain. J. Neurosci. 21: $6718-6731$.

Berghuis, P., Agerman, K., Dobszay, M.B. Minichiello, L., Harkany, T., and Ernfors, P. 2006. Brain-derived neurotrophic factor selectively regulates dendritogenesis of parvalbumin-containing interneurons in the main olfactory bulb through the PLCgamma pathway. J. Neurobiol. 66: 1437-1451.

Chhatwal, J.P., Stanek-Rattiner, L., Davis, M., and Ressler, K.J. 2006. Amygdala BDNF signaling is required for consolidation but not encoding of extinction. Nat. Neurosci. 9: 870-872.

Ennis, M., Linster, C., Aroniadou-Anderjaska, V., Ciombor, K., and Shipley, M.T. 1998. Glutamate and synaptic plasticity at mammalian primary olfactory synapses. Ann. N. Y. Acad. Sci. 855: 457-466.

Fadool, D.A., Tucker, K., Perkins, R., Fasciani, G., Thompson, R.N., Parsons, A.D., Overton, J.M., Koni, P.A., Flavell, R.A., and Kaczmarek, L.K. 2004. Kv1.3 channel gene-targeted deletion produces "Super-Smeller Mice" with altered glomeruli, interacting scaffolding proteins, and biophysics. Neuron 41: 389-404.

Falkenberg, T., Mohammed, A.K., Henriksson, B., Persson, H., Winblad, B., and Lindefors, N. 1992. Increased expression of brain-derived neurotrophic factor mRNA in rat hippocampus is associated with improved spatial memory and enriched environment. Neurosci. Lett. 138: $153-156$.

Fletcher, M.L. and Wilson, D.A. 2003. Olfactory bulb mitral-tufted cell plasticity: Odorant-specific tuning reflects previous odorant exposure. J. Neurosci. 23: 6946-6955.

Funk, D. and Amir, S. 2000. Enhanced Fos expression within the primary olfactory and limbic pathways induced by an aversive conditioned odor stimulus. Neuroscience 98: 403-406.

Hall, J., Thomas, K.L., and Everitt, B.J. 2000. Rapid and selective induction of BDNF expression in the hippocampus during contextual learning. Nat. Neurosci. 3: 533-535.

Heldt, S.A., Stanek, L., Chhatwal, J.P., and Ressler, K.J. 2007. Hippocampus-specific deletion of BDNF in adult mice impairs spatial memory and extinction of aversive memories. Mol. Psychiatry 12: $656-670$. 
Illig, K.R. 2007. Developmental changes in odor-evoked activity in rat piriform cortex. Neuroscience 145: 370-376.

Johnson, D.M., Illig, K.R., Behan, M., and Haberly, L.B. 2000. New features of connectivity in piriform cortex visualized by intracellular injection of pyramidal cells suggest that "primary" olfactory cortex functions like "association" cortex in other sensory systems. $J$. Neurosci. 20: 6974-6982.

Jones, S.V., Heldt, S.A., Davis, M., and Ressler, K.J. 2005. Olfactory-mediated fear conditioning in mice: Simultaneous measurements of fear-potentiated startle and freezing. Behav. Neurosci. 119: 329-335.

Katoh-Semba, R., Takeuchi, I.K., Inaguma, Y., Ito, H., and Kato, K. 1999. Brain-derived neurotrophic factor, nerve growth and neurotrophin-3 selected regions of the rat brain following kainic acid-induced seizure activity. Neurosci. Res. 35: 19-29.

Knafo, S., Grossman, Y., Barkai, E., and Benshalom, G. 2001. Olfactory learning is associated with increased spine density along apical dendrites of pyramidal neurons in the rat piriform cortex. Eur. J. Neurosci. 13: 633-638.

Korte, M., Carroll, P., Wolf, E., Brem, G., Thoenen, H., and Bonhoeffer, T. 1995. Hippocampal long-term potentiation is impaired in mice lacking brain-derived neurotrophic factor. Proc. Natl. Acad. Sci. 92: $8856-8860$.

Lebel, D., Grossman, Y., and Barkai, E. 2001. Olfactory learning modifies predisposition for long-term potentiation and long-term depression induction in the rat piriform (olfactory) cortex. Cereb. Cortex 11: $485-489$.

Lohof, A.M., Ip, N.Y., and Poo, M.M. 1993. Potentiation of developing neuromuscular synapses by the neurotrophins NT-3 and BDNF. Nature 363: 350-353.

Minichiello, L., Calella, A.M., Medina, D.L., Bonhoeffer, T., Klein, R., and Korte, M. 2002. Mechanism of TrkB-mediated hippocampal long-term potentiation. Neuron 36: 121-137.

Mizuno, M., Yamada, K., Olariu, A., Nawa, H., and Nabeshima, T. 2000. Involvement of brain-derived neurotrophic factor in spatial memory formation and maintenance in a radial arm maze test in rats. $J$. Neurosci. 20: 7116-7121.

Nanobashvili, A., Jakubs, K., and Kokaia, M. 2005. Chronic BDNF deficiency permanently modifies excitatory synapses in the piriform cortex. J. Neurosci. Res. 81: 696-705.

Paschall, G.Y. and Davis, M. 2002. Olfactory-mediated fear-potentiated startle. Behav. Neurosci. 116: 4-12.
Rattiner, L.M., Davis, M., and Ressler, K.J. 2004a. Differential regulation of brain-derived neurotrophic factor transcripts during the consolidation of fear learning. Learn. Mem. 11: 727-731.

Rattiner, L.M., Davis, M., French, C.T., and Ressler, K.J. 2004 b. Brain-derived neurotrophic factor and tyrosine kinase receptor B involvement in amygdala-dependent fear conditioning. J. Neurosci. 24: $4796-4806$

Rattiner, L.M., Davis, M., and Ressler, K.J. 2005. Brain-derived neurotrophic factor in amygdala-dependent learning. Neuroscientist 11: $323-333$.

Rex, C.S., Lin, C.Y., Kramar, E.A., Chen, L.Y., Gall, C.M., and Lynch, G. 2007. Brain-derived neurotrophic factor promotes long-term potentiation-related cytoskeletal changes in adult hippocampus. J. Neurosci. 27: 3017-3029.

Richardson, R., Vishney, A., and Lee, J. 1999. Conditioned odor potentiation of startle in rats. Behav. Neurosci. 113: 787-794.

Schettino, L.F. and Otto, T. 2001. Patterns of Fos expression in the amygdala and ventral perirhinal cortex induced by training in an olfactory fear conditioning paradigm. Behav. Neurosci. 115: $1257-1272$.

Tucker, K. and Fadool, D.A. 2002. Neurotrophin modulation of voltage-gated potassium channels in rat through TrkB receptors is time and sensory experience dependent. J. Physiol. 542: 413429.

Tyler, W.J. and Pozzo-Miller, L. 2003. Miniature synaptic transmission and BDNF modulate dendritic spine growth and form in rat CA1 neurones. J. Physiol. 553: 497-509.

Tyler, W.J., Alonso, M., Bramham, C.R., and Pozzo-Miller, L.D. 2002. From acquisition to consolidation: On the role of brain-derived neurotrophic factor signaling in hippocampal-dependent learning. Learn. Mem. 9: 224-237.

Wilson, D.A. 2003. Rapid, experience-induced enhancement in odorant discrimination by anterior piriform cortex neurons. J. Neurophysiol. 90: $65-72$.

Yoshii, A. and Constantine-Paton, M. 2007. BDNF induces transport of PSD-95 to dendrites through PI3K-AKT signaling after NMDA receptor activation. Nat. Neurosci. 10: 702-711.

Received September 19, 2007; accepted in revised form October 26, 2007. 


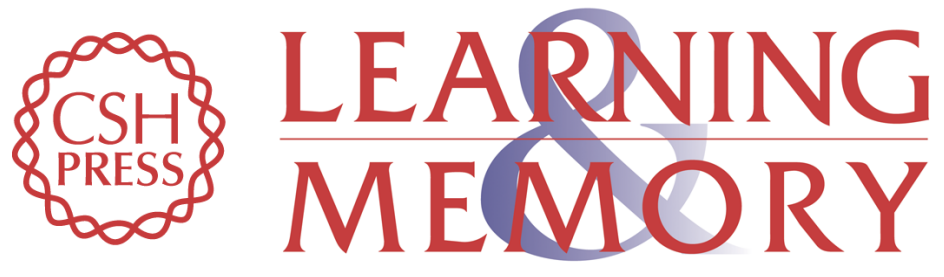

\section{Differential regional expression of brain-derived neurotrophic factor following olfactory fear learning}

Seth V. Jones, Lisa Stanek-Rattiner, Michael Davis, et al.

Learn. Mem. 2007, 14:

Access the most recent version at doi:10.1101//m.781507

References This article cites 33 articles, 9 of which can be accessed free at:

http://learnmem.cshlp.org/content/14/12/816.full.html\#ref-list-1

License

Email Alerting Receive free email alerts when new articles cite this article - sign up in the box at the Service top right corner of the article or click here. 